\title{
Research on Channel Codec of DCR System Based on CMX7141
}

\author{
Yufeng Li ${ }^{1,2}$, Jun Yang ${ }^{1}$, Qingyang Guan ${ }^{1}$ \\ ${ }^{1}$ College of Electronic and Information Engineering, Shenyang aerospace university, Shenyang, China \\ ${ }^{2}$ National Mobile Communications Research Laboratory, Southeast University, Nanjing, China \\ Email: li_yufeng@126.com, yangjun19880520@163.com, 1194392028@qq.com
}

Received July, 2013

\begin{abstract}
This article introduces a digital private network of mobile communication dedicated chipset CMX7141 that used for the 4FSK modulation and demodulation in DCR system, and introduces the application of CMX7141 in the DCR system. Focus research on the channel codec techniques of the chip and have a simulation on the channel coding and decoding. The simulation results indicate that, through channel error correction technique in CMX7141, the BER of DCR system reduces greatly and the transmission quality of DCR system is improved.
\end{abstract}

Keywords: DCR; CMX7141; Cyclic Redundancy Check; Punctured Convolutional Coding

\section{Introduction}

With the development of the information age, people urgently request the intercom have the function such as voice encryption, data transmission, net dispatching and so on. The traditional analog radio defect has been gradually reflected. Private wireless communications system is gradually transform from analog to digital. Currently, digital intercom has received worldwide attention, research on digital intercom technology and the standardized work is prompting forward.

Commonly used international communication standard of digital radio such as: TETRA, DMR[1], dPMR[2] and DCR standard[3], TETRA (Trans European Terrestrial Trunked Radio) Digital trunking communication system is professional mobile communication system based on TDMA digital technology; Digital mobile radio standard was released for professional radio users (PMR) by ETSI; DPMR (digital private mobile radio) is a digital radio protocol, it through provides user the low cost, use lowcomplexity technology realizes the advanced function. ICOM、KENWOOD and many other Japanese manufacturers promulgated DCR standard for digital commercial interphone. It's enacted based on digital Private Mobile Radio (dPMR). Its present standard is ARIB STD98.

CMX7141[4] is the product of CML Semiconductor Company using in the digital special network mobile communication. It's conforming to ARIB's T98 Digital Convenience Radio standard. It implements modulation and demodulation, channel coding and decoding, framing and frame disassembled, and many other key technologies in DCR standard.

Digital interphone working in the wireless environment will inevitably be affected by the variety interferences. Design of channel codec for DCR system has an important impact on the performance. This paper focuses research on codec design in DCR system and punctured convolutional encoding and decoding analyze the BER of different codec mode and gives the Matlab simulation results.

\section{The Introduction of DCR Protocol}

The DCR protocol is a digital handset standard, which was set for commercial digital interphone by Association of Radio Industries and business (ARIB). The DCR protocol has the following characteristics:

- Using frequency division multiple access methods.

- Using the $400 \mathrm{MHz}$ frequency band and the channel spacing is $6.25 \mathrm{kHz}$.

- Half-duplex communications.

- Using 4 FSK modulation and demodulation.

- The coding scheme is CRC [5], convolution codes, interleaving and scrambling.

- The information was transmitted in frame format, the length of a frame of $80 \mathrm{~ms}$ and a super frame consists of 4 frames and so on.

\section{Function and Internal Module Introduction for CMX7141}

CMX7141 is a high integration digital PMR processor. 
CMX7141 is a 4FSK modulation and demodulation chips that compatible with ARIB STD - 98 DCR standard. Much of the ARIB STD - 98 DCR standard air interface protocol is embedded within the CMX7141 operation namely:

Air Interface - Physical Layer 1:

- $\quad$ 4FSK modulation and demodulation

- Bit and symbol definition.

- $\quad$ Frequency and symbol synchronization.

Transmission burst building and splitting.

Air Interface - Data Link Layer 2:

- Channel coding (FEC, CRC).

- Interleaving, de-interleaving and bit ordering.

- $\quad$ Frame building and synchronizing.

- Burst and parameter definition.

- $\quad$ Link addressing (source and destination).

- Interfacing of voice applications (voice data)

with the Physical Layer.

- Data bearer services.

- Exchanging signaling and/or user data with the Call Control Layer.

- Automatic Own-ID detection.

In addition to the above function, CMX7141 has many other functions and Auxiliary Functions.FI (functional image file) defines the functions and features of CMX7141, before use needs to download FI to CMX7141 chip.CMX7141 internal function module shown in Figure 1.

Figure 1 show that the CMX7141 has fully realized the channel coding and decoding technology specified in DCR Protocol, including cyclic redundancy check, Punctured convolutional coding, interweave and scrambling. In addition to channel coding and decoding were completed by CMX7141, frames building and splitting were also done by CMX7141, Two frame types are defined by the standard: "Synchronous Burst 0" (SB0) and "Service Channel" (SC) frames. The two types of frame structure are shown in Figure 2.

"P" indicate preamble, it's some particular symbols' periodic sequence and its length can be defined. "SW" means synchronization word; its length is 20 bits. Both types of frame start with a 20-bit Sync Word, followed by a 16-bit Radio Information Channel (RICH) block and a 60-bit Slow Associated Control Channel (SACCH) block.

RICH can control logical channel identification, communication pattern recognition and work pattern recognition. RICH raw data is 7-bit, insert a parity check at the tail and then handle the data with double bit transform. We can get 16-bit RICH data.

SACCH used to transmit control information, the SACCH raw data is 36-bit.The former 8-bit is an $\mathrm{M}$ sequences and the last 18-bit is control information. Firstly, the SACCH raw data has a CRC (32, 26) processing, insert four bits zero at the end of the data. Then, carries on the punctured convolutional coding and interweave, finally can get 60-bit SACCH data.

SC frames contain two144-bit Traffic Channel (TCH) blocks. These can carry either "speech", uncoded "nonspeech" or FEC coded "non-speech" payload data. TCH raw data is 80-bit.The data generation process is similar to SACCH. The difference is TCH use $\operatorname{CRC}(92,80)$, interweave depth is also different.

SB0 frames also contain a single 144-bit parameter information channel (PICH) block and 144-bit of padding.

The PICH block contains the 36-bit Call Sign (CSM) field, which is the transmitting radio's unique nine-digit serial number encoded in binary-coded decimal (BCD) format. The data generation process is same with TCH.

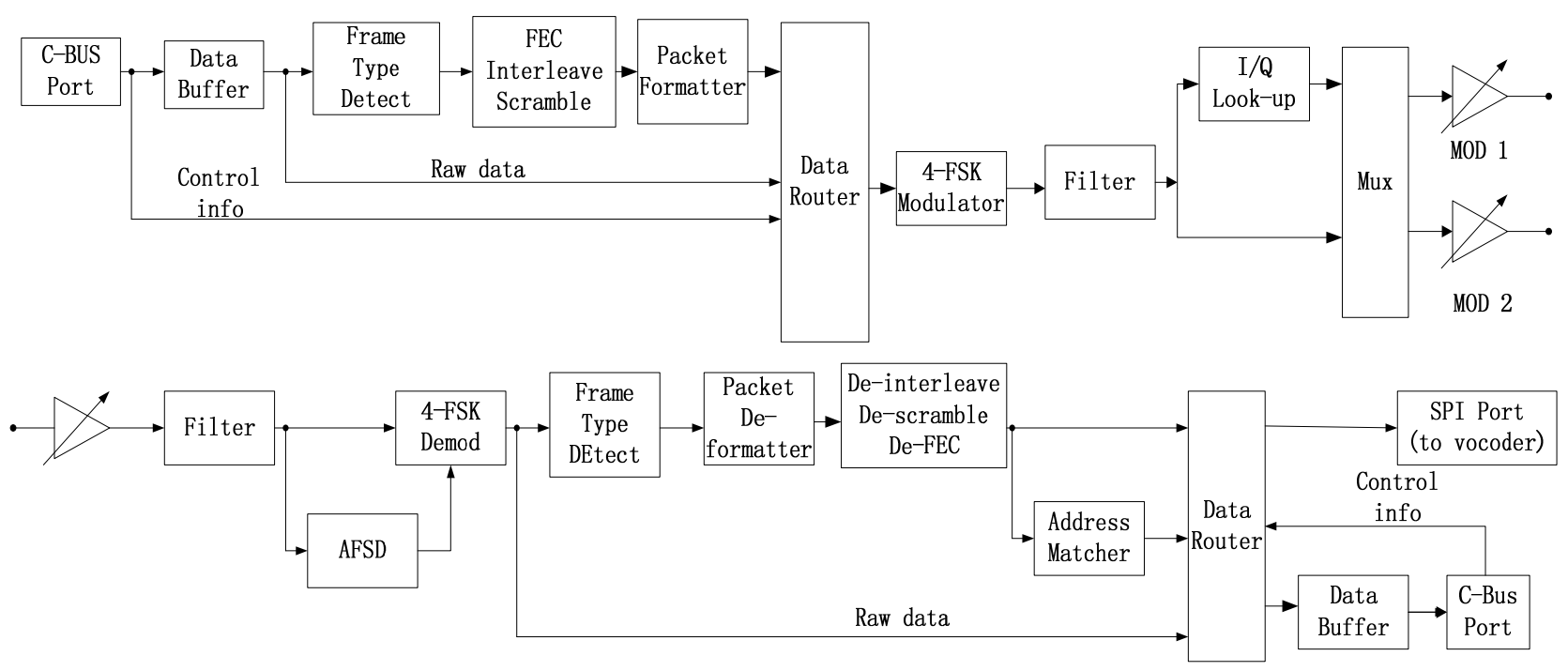

Figure 1. CMX7141 internal module diagram. 


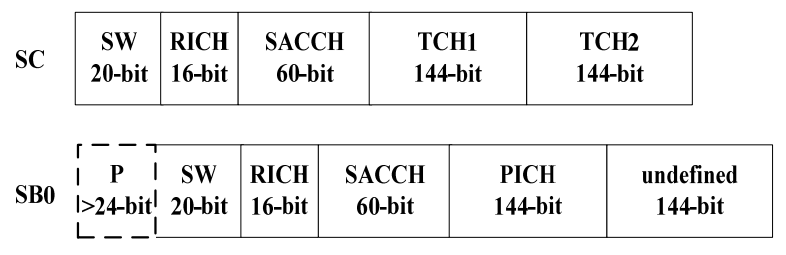

Figure 2. Two kind of frame transfer in CMX7141.

A DCR call start with one (or more) SB0 frames followed by a stream of SC frames carrying payload data. Figure 2 shows that the length of each frame is 384-bits, the duration is $80 \mathrm{~ms}$.Four frames can compose a super frame.CMX7141 can achieve frame synchronization automatically.

\section{Channel Coding and Decoding Technology in CMX7141}

\subsection{CRC}

Cyclic Redundancy Check (CRC) code is a common type of error checking code. The error detection capability is as follows:

- Burst error length $\leq n-k$.

- Majority of burst error length $=n-k+1$, the proportion of such errors that cannot be detected is $2^{-(n-k)}$.

- Majority of burst error length $>n-k$, the ratio of the error cannot be detect is $2^{-(n-k)}$.

- The error of all the code distance of the allow code group $\leq d_{\min }-1$.

- All odd number of errors.

The two kind of CRC are CRC-6 and CRC-12 in the DCR standard. The expression of cyclic redundancy check code is (n, k). "n" indicate the length of the encoded; " $\mathrm{k}$ " means the length of the original information. CRC coding processing is:

$$
\frac{x^{n-k} m(x)}{g(x)}=Q(x)+\frac{r(x)}{g(x)}
$$

In this formula, " $\mathrm{m}(\mathrm{x})$ " represent the code polynomial, "g(x)" represent the generate polynomial, " $\mathrm{Q}(\mathrm{x})$ " is quotient and " $\mathrm{r}(\mathrm{x}) / \mathrm{g}(\mathrm{x})$ " is the remainder. The remainder is also a polynomial, and then we can get the coding is:

$$
C(x)=x^{n-k} m(x)+b(x)
$$

CRC was widely used in data storage and data communication, making in the disk driver and the correspondence product (such as modulator and demodulator) in hardware form. Its hardware implementation is shown in Figure 3.

The decoding process of CRC is: (1) Using the receiving code polynomial $r(x)$ divided by generate polynomial $g(x)$ and obtain adjoin polynomial $s(x) ;(2)$ According to $s(x)$ to determine the error pattern $e(x)$; (3) $r(x) \oplus g(x)$, Correct the mistakes.

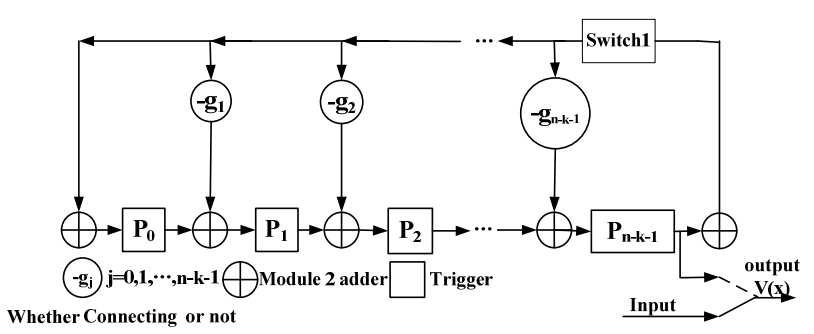

Figure 3. (n-k) level feedback shift register code electric circuit.

\subsection{Punctured Convolutional Coding}

If the system uses $(n, 1, m)$ convolutional coding, the maximum rate we can obtain is $1 / 2$. For many applications, they need a higher coding rate. This can be achieved by punctured convolutional[6-8] code. Puncturing process in fact is deletes part of elements in the encoder output symbol stream, the number of deleted symbols determining the final coding rate. The main advantage is using the same encoder, by changing the delete code number can make a wide range of different in coding rate. In the realization of puncturing, it must determine puncturing pattern. " $\mathrm{P}$ " is a puncturing matrix and it's an $n \times p$ order matrix.

1) The coding of Punctured convolution code

In DCR protocol, using the punctured convolutional code of $(2,1,5)$ convolutional code. The generating polynomial is:

$$
\begin{aligned}
& G_{1}(D)=1+D^{3}+D^{4} \\
& G_{2}(D)=1+D+D^{2}+D^{4}
\end{aligned}
$$

The output information according to order of $G_{1}, G_{2}$, they are alternating readout.

Take the SACCH channel data as an example, the raw data is 36-bit, Supposes the encoder initial state is zero. Along with time $t$ recursion and information

$$
\left(m_{0}, m_{1}, m_{2} \ldots \ldots . m_{33}, m_{34}, m_{35}\right)
$$

input constantly, code $\left(C^{0}, C^{1}, C^{2}, C^{3} \ldots \ldots.\right)$ output continuously.

At the time $t=0, C^{0}=m_{0} A^{0}=\left(G_{1}^{0} G_{2}^{0}\right)$

At the time $t=1, C^{1}=m_{1} A^{0}+m_{0} A^{1}=\left(G_{1}^{1} G_{2}^{1}\right)$

At the time $t=2, \quad C^{2}=m_{2} A^{0}+m_{1} A^{1}+m_{0} A^{3}=\left(G_{1}^{2} G_{2}^{2}\right)$

At the time $t=3$,

$$
C^{3}=m_{3} A^{0}+m_{2} A^{1}+m_{1} A^{2}+m_{0} A^{3}=\left(G_{1}^{3} G_{2}^{3}\right)
$$

At the time $t=4$,

$$
C^{4}=m_{4} A^{0}+m_{3} A^{1}+m_{2} A^{2}+m_{1} A^{3}+m_{0} A^{4}=\left(G_{1}^{4} G_{2}^{4}\right)
$$

At the time $t=5$,

$$
C^{5}=m_{5} A^{0}+m_{4} A^{1}+m_{3} A^{2}+m_{2} A^{3}+m_{1} A^{4}=\left(G_{1}^{5} G_{2}^{5}\right)
$$

Written in matrix form

$$
C=\left(C_{0}, C_{1}, C_{2} \ldots \ldots C_{34}, C_{35}\right)=m_{1 \times 36} A_{36 \times 36}
$$




$$
\begin{gathered}
=\left(m_{0}, m_{1}, m_{2} \ldots \ldots m_{34}, m_{35}\right) \\
\times\left[\begin{array}{ccccccccc}
A^{0} & A^{1} & A^{2} & A^{3} & A^{4} & \ldots & \ldots & 0 \\
0 & A^{0} & A^{1} & A^{2} & A^{3} & A^{4} & \ldots & 0 \\
0 & 0 & A^{0} & A^{1} & A^{2} & A^{3} & \ldots & 0 \\
0 & 0 & 0 & A^{0} & A^{1} & A^{2} & \ldots & 0 \\
\ldots & \ldots & \ldots & \ldots & \ldots & \ldots & \ldots & \ldots \\
0 & 0 & 0 & 0 & 0 & 0 & \ldots & A^{4}
\end{array}\right]
\end{gathered}
$$

$G_{1}^{t} G_{2}^{t}$ Represents the output at the time $t, A^{j}$ Represents the matrix coefficient.

We can obtain the coefficient matrix by the generate polynomial:

$$
A^{0}=\left(\begin{array}{ll}
1 & 1
\end{array}\right), A^{1}=\left(\begin{array}{ll}
0 & 1
\end{array}\right), A^{2}=\left(\begin{array}{ll}
0 & 1
\end{array}\right), A^{3}=\left(\begin{array}{ll}
1 & 0
\end{array}\right), A^{4}=(1
$$

After convolution encoding, the data from 36-bit becomes 72-bit.

Then puncturing, for different traffic channel provides a different puncturing pattern. The puncturing pattern of SACCH channel data is:

$$
P=\left(\begin{array}{llllll}
1 & 1 & 1 & 1 & 1 & 1 \\
1 & 1 & 0 & 1 & 1 & 0
\end{array}\right)
$$

And the TCH/PICH data puncturing pattern is:

$$
P=\left(\begin{array}{ll}
1 & 1 \\
0 & 1
\end{array}\right)
$$

Take the TCH channel data as the example, Convolution encoder output:

$$
C=\left(C_{0}^{(1)}, C_{0}^{(2)}, C_{1}^{(1)}, C_{1}^{(2)}, C_{2}^{(1)}, C_{2}^{(2)}, C_{3}^{(1)}, C_{3}^{(2)}, \ldots \ldots\right)
$$

After puncturing process, the output is:

$$
C=\left(C_{0}^{(1)}, C_{1}^{(1)}, C_{1}^{(2)}, C_{2}^{(1)}, C_{3}^{(1)}, \ldots \ldots\right)
$$

Punctured convolution codes can implement different rate requirements, And in the premise of ensuring the reliability, can improve the transmission efficiency. And because the codec is relatively simple and the error correction performance is good, therefore it is widely used in channel error correction.

2) The decoding of Punctured convolution code

The convolution code decoding mainly has the algebra decoding and the probabilistic decoding. The algebra decoding uses the majority-logic decoding method, the probabilistic decoding divides into the Viterbi decoding and the sequential decoding. The Viterbi decoding algorithm is a maximum-likelihood decoding algorithm that makes the probability of decoding error minimum. It's the optimal algorithm. Viterbi decoding algorithm can divide into the soft decision and the hard decision decoding.

Punctured convolution codes using Viterbi decoder for decoding has the two major ways: a) Zero padding method in the puncturing positions.

b) According to the bit rate of equivalent convolution code to decoding after puncturing.

The second method needs large computations as the request of coding efficiency to be high, the hardware realization complex and the versatility is bad. Currently, usually uses the first method for decoding, zero padding in the puncturing positions and then use the Viterbi decoding.

Convolution code sequence $\mathrm{C}$ is the convolution of input sequences $\mathrm{m}$ and impulse response g. Convolution code sequence $\mathrm{C}$ after signaling mapping result is y. After modulation through the noise channel, Arrive at the receiving end receives the sequence $r$. Viterbi decoding algorithm is using the receive sequence $r$, according to the maximum likelihood criterion to estimate the code sequence y. The decoding algorithm can be implemented as follows:

a) Zero padding method in the puncturing positions.

b) The $S_{k, t}$ indicates the State $S_{k}$ at the time of $t$ in the trellis diagram, specify a metric value $V\left(S_{k, t}\right)$ to each state in the trellis diagram and initialization:at the time $t=0, V\left(S_{0,0}\right)=0$, other state $V\left(S_{k, 0}\right)=+\infty$.

c) $t+1 \rightarrow t$, Calculate measurement value of the entire path to the state $S_{k}$ in the time $t$. Firstly calculate branch metric at $t: M\left(r_{t} \mid y_{t}\right)=\sum_{j=1}^{n_{0}} M\left(r_{t}^{(j)} \mid y_{t}^{(j)}\right)$, if use soft decision through calculate the square of Euclidean distance $\sum_{j=1}^{n_{0}}\left(r_{t}^{(j)}-y_{t}^{(j)}\right)^{2}$, hard decision use hamming distance $\sum_{j=1}^{n_{0}}\left|\left(r_{t}^{(j)}-y_{t}^{(j)}\right)\right|$. Then calculates metric value of the partial path at time $t: M^{t}(r \mid y)=\sum^{t} M\left(r_{i} \mid y_{i}\right)$, through calculates $V\left(S_{k, t}\right)+M\left(r_{t} \mid y_{t}\right)$ to réalize.

d) Set the metric value $V\left(S_{k, t}\right)$ as the best path to the state $S_{k}$ at time $t$. If have more than one best part of path metric, we can select any one of these.

e) Store the best part of the survivor path metric and the corresponding status and path.

f) If $t<L+m-1$, returns to the second step, Otherwise read-out surviving path, the corresponding mark is the decoding output.

The Viterbi algorithm finally obtains the surviving path in the trellis diagram is unique. That is also the maximum likelihood path.

\subsection{Interleaving}

The template is designed so that author affiliations are not repeated each time for multiple authors of the same affiliation. Please keep your affiliations as succinct as possible (for example, do NOT post your job titles, positions, academic degrees, zip codes, names of build- 
ing/street/district/province/state, etc.). This template was designed for two affiliations.

1) For author/s of only one affiliation: To change the default, adjust the template as follows.

a) Selection: Highlight all author and affiliation lines.

b) Change number of columns: Select the Columns icon from the MS Word Standard toolbar and then select "1 Column" from the selection palette.

c) Deletion: Delete the author and affiliation lines for the second affiliation.

2) For author/s of more than two affiliations: To change the default, adjust the template as follows.

a) Selection: Highlight all author and affiliation lines.

b) Change number of columns: Select the "Columns" icon from the MS Word Standard toolbar and then select "1 Column" from the selection palette.

c) Highlight author and affiliation lines of affiliation 1 and copy this selection.

d) Formatting: Insert one hard return immediately after the last character of the last affiliation line. Then paste down the copy of affiliation 1. Repeat as necessary for each additional affiliation.

\section{The Simulation Results}

The random signal source produces the binary random sequence, after channel coding and interweaves carries on the 4FSK modulation, through the multipath fading channel. Then, carries on the demodulation, de-interleave and channel decoding, use MATLAB simulate this process, and draws Non-error correction channel BER curve and Error correction coding channel BER curves:

Figure 4 shows that: Error correction coding channel BER is much lower than the Non-error correction code channel BER. And when the signal-to-noise ratio is same,

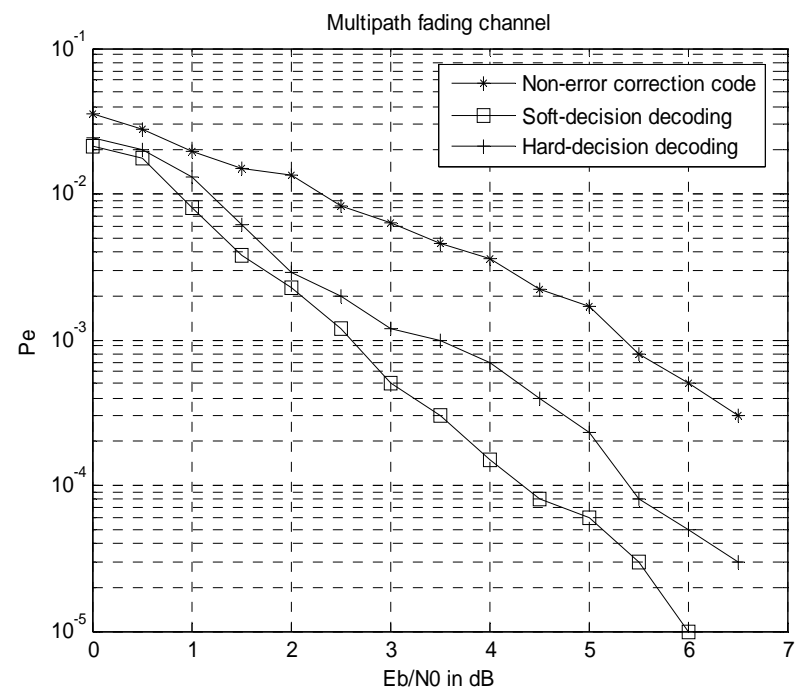

Figure 4. The error correction performance of punctured convolution code.
Soft-decision decoding is better than the hard-decision decoding. Moreover CMX7141 can select soft decision or hard decision according to the needs of users. This ensures error-correcting performance of error-correcting code. Thereby channel coding can improve the quality of voice communication.

\section{Conclusions}

This article introduces a dedicated chipset CMX7141 that apply to DCR standard. And in conjunction with DCR standard analysis CMX7141 internal algorithm and simulate these algorithms. Enable the people to have certain understanding to the digital intercom key technologies, it's convenient for people to improve algorithm on the basis of the original algorithm and provide a theoretical foundation for new intercom standard, at the same time to promote the development of digital radio technology.

\section{Acknowledgements}

This article was supported by the National Natural Science Foundation contract NSFC No.61171081, the Jiangsu Postdoctoral Sustentation Fund contract No. 1101077C and the Fund of Aeronautics Science contract No. 20122654004.

\section{REFERENCES}

[1] ETSITR 102 398V1.1.2 (2008-05). Electromagnetic Compatibility and Radio Spectrum Matters (ERM); Digital Mobile Radio (DMR) General System Design. ETSI, 2008.

[2] ETSITR 102 490V1.2.1 (2006-08). Electromagnetic compatibility and Radio Spectrum Matters (ERM); Peer-to-Peer Digital Private Mobile Radio (DPMR) using FDMA with a Channel Spacing of 6,25 kHz with e.r.p. of up to $500 \mathrm{~mW}$. ETSI, 2006.

[3] Digital Convenience Radio Equipment for Simplified Service V.1.3 Association of Radio Industries and Businesses.

2012.12.18.http://www.arib.or.jp/english/html/overview/d oc/1-STD-T98v1_3.pdf.

[4] CML Microcircuits. Datasheet of CMX7131/CMX7141 Digital PMR Processor DCR Operation. CML Microcircuits, 2011.

[5] R. Prevost, M. Coulon, D. Bonacci, et al., "CRC-assisted Error Correction in a Trellis Coded System with Bit Stuffing," Statistical Signal Processing Workshop (SSP), 2011 IEEE. Nice: SSP, 2011.pp. 381-384.

[6] B. F. Uchoa-Filho, D. R. Souza, C. Pimentel, et al., "Generalized Punctured Convolutional Codes,” Communications Letters, IEEE. Sep. 2005. pp. 1070-1072.

[7] J. Geldmacher and J. Goetze "Syndrome Former Trellis Construction for Punctured Convolutional Codes," Information Theory and its Applications (ISITA), 2010 In- 
ternational Symposium on. Taichung: ISITA. 2010. pp.478-483.

[8] A. Katsiotis and N. Kalouptsidis, “On (n,n-1) Punctured
Convolutional Codes and Their Trellis Modules,” Communications, IEEE Transactions on, 2011, pp. 1213-1217. 